\title{
ALMOST PERIODIC HOMOGENIZATION OF THE KLEIN-GORDON TYPE EQUATION
}

\author{
LAZARUS SIGNING
}

Abstract. In this paper, the homogenization problem for the Klein-Gordon type equation is studied in the almost periodic setting. The propagation speed and the potential are spatial and time dependent almost periodically varying functions. One convergence theorem is proved and we derive the macroscopic homogenized model verified by the mean wave function.

Mathematics subject classification (2010): 35B27, 35B40, 81Q05.

Keywords and phrases: Homogenization, Klein-Gordon type equation, sigma-convergence.

\section{REFERENCES}

[1] A. Bensoussan, J.L. Lions and G. Papanicolaou, Asymptotic Analysis for Periodic Structures, North-Holland, 1978.

[2] A.S. Besicovitch, Almost Periodic Functions, Cambridge at the University Press, Dover Publications, Inc. 1954

[3] N. Bourbaki, Intégration, Chap. 1-4, Hermaan, Paris, 1966.

[4] N. Bourbaki, Topologie générale, Chap. 5-10, Hermaan, Paris, 1974.

[5] S. Brahim-Otsmane, G.A. Francfort And F. Murat, Correctors for the homogenization of the wave and heat equations, J. Math. Pures Appl.,71 (1992), p. 197-231.

[6] D. Cioranescu, P. Donato, An Introduction to Homogenization, Oxford lecture series in Mathematics and Its Applications; 17 (1999).

[7] J. J. F. Fournier, J. Stewart, Amalgams of $L^{p}$ and $l^{q}$, Bull. Amer. Math. Soc. 13 (1985), p. 1-21.

[8] A. Guichardet, Analyse Harmonique commutative. Dunod, Paris, 1968.

[9] R. LARSEN, Banach Algebras, Marcel Dekker, New York, 1973.

[10] J. L. Lions, Contrôle Optimal de Systèmes Gouvernés par les Equations aux Dérivées Partielles, Dunod Gauthier-Villars, Paris 1968.

[11] J.L. Lions, E. Magenes, Problèmes aux Limites non homogènes et Applications 2, Dunod, Paris 1968.

[12] D. Luk kassen, G. Nguetseng and P. Wall, Two-scale convergence, Int. J. Pure and Appl. Math, 2 (2002), 35-86.

[13] G. NGUETSENG, A general convergence result for a functional related to the theory of homogenization, SIAM J. Math. Anal., 20 (1989), 608-623.

[14] G. Nguetseng, Almost periodic homogenization: Asymptotic analysis of a second order elliptic equation, (Publ. math. LAN 01). Univ. Yde I 2000.

[15] G. NGUetseng, Deterministic homogenization of a semilinear elliptic partial differential equation of order 2m, Maths, Reports, 8 (58) (2006), 167-195.

[16] G. Nguetseng, Sigma-convergence of parabolic differential operators, Multiscale problems in biomathematics, mechanics, physics and numerics, 93-132, Gakuto Internat. Ser. Math. Sci. Appl., 31, Tokyo, 2009.

[17] G. Nguetseng, Homogenization Structures and applications I, Zeit. Anal. Anwend. 22 (2003) $73-$ 107.

[18] G. Nguetseng And H. NnAng, Homogenization of Nonlinear monotone operators beyond the periodic setting, Electronic Journal of Differential Equations, Vol. 2003 (2003), No. 36, pp. 1-24. 
[19] G. Nguetseng And N. Svanstedt, $\Sigma$-convergence, Banach J. Math. Anal. 5 (2011), No. 1, 101135.

[20] G. Nguetseng And J.L. Woukeng, Deterministic homogenization of parabolic monotone operators with time dependent coefficients, Electronic Journal of Differential Equations, Vol. 2004 (2004), No. 82, pp. 1-23. 